\title{
DISTURBANCE DECOUPLING WITH STABILITY IN DISCRETE-TIME SWITCHING LINEAR SYSTEMS: ARBITRARY SWITCHING
}

\author{
Elena Zattoni \\ Department of Electrical, Electronic, and \\ Information Engineering "Guglielmo Marconi" \\ Alma Mater Studiorum · University of Bologna \\ Viale Risorgimento 2, 40136 Bologna, ITALY
}

\begin{abstract}
The problem of disturbance decoupling with stability consists in finding a compensator that renders the output of a dynamical system insensitive to undesired inputs, while assuring stability, in a suitable sense, of the compensated dynamics. Disturbance decoupling is a classic problem in control theory as well as a primary concern in control system design. This work deals with disturbance decoupling in discrete-time switching linear systems subject to arbitrary switching. Inaccessible and measurable disturbances are considered in a unified setting. Different problem formulations are investigated, with progressively more severe stability requirements: namely, the study starts from structural decoupling and ends to decoupling with input-to-state stability. Solvability conditions for the stated problems are proven. In particular, necessary conditions for stabilization of the inner and outer switching dynamics of the resolving subspace are shown. A convex procedure for the computation of the switching state feedback which simultaneously achieves disturbance decoupling and closed-loop stability is presented. The proposed methodology is illustrated through a worked out numerical example.
\end{abstract}

AMS Subject Classification: 93C30, 93C 35, 93C55

Key Words: switching systems, multivariable systems, discrete-time systems

Received: $\quad$ August 6, 2016

Revised: $\quad$ October 20, 2016

Published: October 21, 2016 (c) 2016 Academic Publications, Ltd. url: www.acadpubl.eu 


\section{Introduction}

The problem of disturbance decoupling was first solved for linear time-invariant systems in the framework of the geometric approach [1,2]. Various formulations of the problem, depending on the information available on the to-be-decoupled signals have also been considered in the same framework for linear systems $[3,4,5,6]$. Disturbance decoupling has been studied for various other classes of dynamical systems and, because of the peculiarities arising in each context, it still attracts the interest of the research community. Indeed, disturbance decoupling has recently been considered for nonlinear systems [7], descriptor systems and systems over rings [8], time-delay systems [9, 10], linear parameter varying systems [11, 12], hybrid linear systems with state jumps [13, 14], and switching linear systems $[15,16,17,18,19,20,21,22,23,24,25,26,27]$. The motivation for investigating possible solutions of the disturbance decoupling problem for more general classes of dynamical systems than linear time-invariant systems is not only the intrinsic theoretic interest of finding relaxed solvability conditions, but also the need to find powerful tools to solve more intricate problems like, for instance, model matching [28, 29, 30, 31].

As to disturbance decoupling in switching linear systems, the complexity of such dynamical systems gives rise to a variety of problem formulations. In fact, switching linear systems - which are the object of a wide literature (see, e.g., the books $[32,33,34])$ - consist of a set of linear time-invariant systems, the so-called modes, and a signal designating the active mode at each instant of time, the switching signal. Therefore, many approaches are possible depending on several aspects: for instance, i) the manipulable variables (namely, the switching signal, the control input, or both); ii) the type of the admissible switching signals (i.e., arbitrary or constrained ones); iii) the stability requisites for the compensated switching system (i.e., quadratic, exponential, or asymptotic stability).

Concerning the manipulable variables, in $[15,17]$ the manipulable variable is the control input, while the switching signal is regarded as a disturbance the same way as the exogenous input. In $[23,16]$ both the switching signal and the control input are to be designed so as to achieve decoupling of the exogenous disturbance. In $[18,19,20,21,22,24,25,26,27]$, the manipulable variable is the control input, but the switching signal is assumed to be measurable hence, available to the compensator.

As mentioned above, another relevant distinction concerns the admissible class of switching signals. In [21, 22, 26, 27], the switching signals are assumed to be subject to a constraint on the minimum dwell-time: i.e., the length of the 
time interval between two consecutive switches must be greater than or equal to a positive real constant. In $[18,19,20,24,25]$, the admissible switching signals are assumed to be arbitrary, under the only provision that the number of switches in any finite time interval is finite, so as to avoid possible Zeno behaviors.

Strictly related to the nature of the admitted switching signals, there is the stability requisite on the compensated switching system. Altough some of the abovementioned papers do not take stability into consideration - this is the case of $[15,17]$ — the others can be roughly classified as follows. In [23, 16], quadratic stability is sought for a suitable choice of the switching signal. In $[18,19,20,24,25]$, quadratic stability is required for all the switching signals satisfying the only constraint that the number of switches they cause is finite in any finite time interval. In [21, 22, 26, 27], asymptotic stability is demanded for all the switching signals in a class characterized by a minimum dwell-time.

In particular, this work addresses switching linear systems subject to arbitrary switching and the stability requirement is that of quadratic stability of the compensated switching linear system, like in [18, 19, 20, 24, 25]. However, this work differs from the previous ones in several aspects and it introduces new contributions as explained below. In the first place, [18, 19] are focused on decoupling of disturbances accessible for measurement — in discrete-time and continuous-time, respectively - and present solutions based on switching dynamic feedforward compensators. Meanwhile, [20, 24] consider decoupling of inaccessible signals in the discrete-time case and propose switching state feedback solutions - for systems without the feedthrough term from the control input to the output and, respectively, for systems with such feethrough term. Instead, this work, like [25], deals with decoupling of measurable and inaccessible disturbance in a unified framework, on the basis of a compensation scheme which includes a switching state feedback and a switching algebraic feedward action.

Nonetheless, the most relevant aspect that distinguishes this work from $[18,19,20]$ is considering not only (zero-input) state stability, but also the socalled input-to-state stability [35]. In fact, as pointed out, e.g., in [34], switching linear systems enjoying some zero-input state stability properties are not guaranteed to have good input-to-state stability properties, such as bounded inputs resulting in bounded state trajectories or inputs converging to zero resulting in state trajectories converging to zero. Nonetheless, quadratic stability — which is a conservative requisite with respect, e.g., to asymptotic stability - has the advantage of assuring, at the least, local input-to-state stability [36].

For these reasons, this work takes input-to-state stability into consideration, 
in connection with quadratic stability under arbitrary switching. However, in comparison with $[24,25]$ — which also consider input-to-state stability — the discussion on quadratic stability under arbitrary switching is deepened and specific new results are presented. In particular, this work provides necessary conditions for quadratic stabilizability under arbitrary switching of the inner and outer switching dynamics associated to the maximal robust controlled invariant subspace contained in the null space of the plant output. Such subspace plays a key role in the conditions for solvability of the disturbance decoupling problem and the abovementioned conditions are crucial to check on the existence of a solution based on that maximal subspace. In addition, this work gives prominence to the computational procedure for the synthesis of the switching compensator and illustrates each step through a worked-out non-trivial numerical example.

This work is organized as follows. Sections 2 and 3 introduce the methodological tools - namely, the further elaboration upon notions of the geometric approach needed to deal with switching linear systems. Section 4 deals with structural disturbance decoupling. Section 5 considers disturbance decoupling with stability. Section 7 shows a numerical example. Section 8 collects the concluding remarks.

Notation: $\mathbb{Z}^{+}, \mathbb{R}$, and $\mathbb{C}^{\odot}$ stand for the sets of nonnegative integer numbers, real numbers, and complex numbers with module smaller than 1 , respectively. Matrices and linear maps are denoted by slanted upper-case letters, like $A$. The image, the kernel, and the transpose of $A$ are denoted by $\operatorname{Im} A, \operatorname{Ker} A$, and $A^{\top}$, respectively. The spectrum of a square matrix $A$ is denoted by $\lambda(A)$. Vector spaces and subspaces are denoted by calligraphic letters, like $\mathcal{V}$. The quotient space of a vector space $\mathcal{V}$ over a subspace $\mathcal{W} \subseteq \mathcal{V}$ is denoted by $\mathcal{V} / \mathcal{W}$. The restriction of a linear map $A$ to an $A$-invariant subspace $\mathcal{J}$ is denoted by $\left.A\right|_{\mathcal{J}}$. The inverse image of a subspace $\mathcal{V}$ through a linear map $B$ is denoted by $B^{-1} \mathcal{V}$ (note that $B$ is not necessarily invertible). The inverse of a nonsingular square matrix $T$ is denoted by $T^{-1}$. The dimension of a subspace $\mathcal{V}$ is denoted by $\operatorname{dim} \mathcal{V}$. The symbol $I$ stands for an identity matrix of appropriate dimension. The symbol $\uplus$ is used for union with repetition count.

\section{Geometric Approach to Arbitrary Switching Linear Systems - Structure}

The aim of this section is to introduce the structural geometric notions needed to deal with switching linear systems subject to arbitrary switching — some 
of these notions were first presented for parameter-dependent linear systems in [37]. The discrete-time switching linear system $\Sigma_{\sigma_{t}}$ is defined as

$$
\Sigma_{\sigma_{t}} \equiv\left\{\begin{aligned}
x_{t+1} & =A_{\sigma_{t}} x_{t}+B_{\sigma_{t}} u_{t}+H_{\sigma_{t}} h_{t}, \\
y_{t} & =C_{\sigma_{t}} x_{t},
\end{aligned}\right.
$$

where $t \in \mathbb{Z}^{+}$is the time variable, $x \in \mathcal{X}=\mathbb{R}^{n}$ is the state, $u \in \mathbb{R}^{p}$ is the control input, $h \in \mathbb{R}^{m}$ is the disturbance input, and $y \in \mathbb{R}^{q}$ is the output, with $p, m, q \leq n$. The switching signal $\sigma_{t}$ is defined as the arbitrary, measurable and not a-priori known correspondence $\sigma: \mathbb{Z}^{+} \rightarrow \mathcal{I}$, where $\mathcal{I}=\{1,2, \ldots, N\}$ is the finite index set of the modes of $\Sigma_{\sigma_{t}}$. With a slight abuse of notation, the set of the modes of $\Sigma_{\sigma_{t}}$, which are linear time-invariant systems whose input and output distribution matrices are assumed to be full-rank, is denoted by $\left\{\Sigma_{\sigma}, \sigma \in \mathcal{I}\right\}$.

The symbols $\mathcal{B}_{\sigma}, \mathcal{H}_{\sigma}$, and $\mathcal{C}_{\sigma}$ respectively stand for $\operatorname{Im} B_{\sigma}, \operatorname{Im} H_{\sigma}$, and $\operatorname{Ker} C_{\sigma}$, with $\sigma \in \mathcal{I}$. The symbol $\mathcal{C}$ stands for $\bigcap_{\sigma \in \mathcal{I}} \mathcal{C}_{\sigma}$. A subspace $\mathcal{J} \subseteq \mathcal{X}$ is said to be a robust $A_{\sigma}$-invariant subspace if $A_{\sigma} \mathcal{J} \subseteq \mathcal{J}$ for all $\sigma \in \mathcal{I}$. A subspace $\mathcal{V} \subseteq \mathcal{X}$ is said to be a robust $\left(A_{\sigma}, \mathcal{B}_{\sigma}\right)$-controlled invariant subspace if $A_{\sigma} \mathcal{V} \subseteq \mathcal{V}+\mathcal{B}_{\sigma}$ for all $\sigma \in \mathcal{I}$. Moreover, a subspace $\mathcal{V} \subseteq \mathcal{X}$ is a robust $\left(A_{\sigma}, \mathcal{B}_{\sigma}\right)$ controlled invariant subspace if and only if a set $\left\{F_{\sigma}: \mathbb{R}^{p} \rightarrow \mathbb{R}^{n}, \sigma \in \mathcal{I}\right\}$ exists, such that $\left(A_{\sigma}+B_{\sigma} F_{\sigma}\right) \mathcal{V} \subseteq \mathcal{V}$ for all $\sigma \in \mathcal{I}$. Any such set of linear maps is called a friend of $\mathcal{V}$. The same name is also used to address the associated switching state feedback $F_{\sigma_{t}}$. The set of all robust $\left(A_{\sigma}, \mathcal{B}_{\sigma}\right)$-controlled invariant subspaces contained in a given subspace is an upper semilattice with respect to the sum and the inclusion of subspaces. In particular, the maximal robust $\left(A_{\sigma}, \mathcal{B}_{\sigma}\right)$ controlled invariant subspace contained in $\mathcal{C}$ is denoted by $\mathcal{V}_{R}^{*}$. The subspace $\mathcal{V}_{R}^{*}$, which, as will be shown in the remainder of the work, plays a key role in the solution of the disturbance decoupling problem, can be computed with Algorithm 1 in [37].

The following lemma - whose proof is omitted since it is a matter of simple linear algebra - is aimed at introducing the notions of inner and outer switching dynamics associated to the subspace $\mathcal{V}_{R}^{*}$ by a friend $F_{\sigma_{t}}$.

Lemma 1. Consider the modes $\left\{\Sigma_{\sigma}, \sigma \in \mathcal{I}\right\}$, the subspace $\mathcal{V}_{R}^{*}$, and a friend $\left\{F_{\sigma}, \sigma \in \mathcal{I}\right\}$. Perform a state space basis transformation $T=\left[\begin{array}{ll}T_{1} T_{2}\end{array}\right]$, where $\operatorname{Im} T_{1}=\mathcal{V}_{R}^{*}$. Then, with respect to the new coordinates,

$$
\begin{gathered}
A_{\sigma}^{*}+B_{\sigma}^{*} F_{\sigma}^{*}=T^{-1}\left(A_{\sigma}+B_{\sigma} F_{\sigma}\right) T= \\
{\left[\begin{array}{cc}
A_{11, \sigma}^{*}+B_{1, \sigma}^{*} F_{1, \sigma}^{*} & A_{12, \sigma}^{*}+B_{1, \sigma}^{*} F_{2, \sigma}^{*} \\
0 & A_{22, \sigma}^{*}+B_{2, \sigma}^{*} F_{2, \sigma}^{*}
\end{array}\right],} \\
B_{\sigma}^{*}=T^{-1} B_{\sigma}=\left[\begin{array}{c}
B_{1, \sigma}^{*} \\
B_{2, \sigma}^{*}
\end{array}\right], H_{\sigma}^{*}=T^{-1} H_{\sigma}=\left[\begin{array}{c}
H_{1, \sigma}^{*} \\
H_{2, \sigma}^{*}
\end{array}\right],
\end{gathered}
$$




$$
C_{\sigma}^{*}=C_{\sigma} T=\left[\begin{array}{ll}
0 & C_{2, \sigma}^{*}
\end{array}\right],
$$

for all $\sigma \in \mathcal{I}$.

The structural zero in $A_{\sigma}^{*}+B_{\sigma}^{*} F_{\sigma}^{*}$, holding for all $\sigma \in \mathcal{I}$, shows that the switching dynamics $A_{\sigma_{t}}+B_{\sigma_{t}} F_{\sigma_{t}}$ defines a switching dynamics restricted to the subspace $\mathcal{V}_{R}^{*}$ - denoted by $\left.\left(A_{\sigma_{t}}+B_{\sigma_{t}} F_{\sigma_{t}}\right)\right|_{\mathcal{V}_{R}^{*}}$ - and a switching dynamics induced on the quotient space $\mathcal{X} / \mathcal{V}_{R}^{*}$ - denoted by $\left.\left(A_{\sigma_{t}}+B_{\sigma_{t}} F_{\sigma_{t}}\right)\right|_{\mathcal{X} / \mathcal{V}_{R}^{*}}$. The former switching dynamics is called the inner switching dynamics associated to $\mathcal{V}_{R}^{*}$ by $F_{\sigma_{t}}$, the latter is called the outer switching dynamics associated to $\mathcal{V}_{R}^{*}$ by $F_{\sigma_{t}}$.

\section{Geometric Approach to Arbitrary Switching Linear Systems - Stability}

The purpose of this section is to introduce the notions of inner and outer quadratic stabilizability of $\mathcal{V}_{R}^{*}$ and to provide necessary conditions for $\mathcal{V}_{R}^{*}$ to be inner quadratically stabilizable and outer quadratically stabilizable, respectively, under arbitrary switching.

\subsection{Outer Quadratic Stabilizability of $\mathcal{V}_{R}^{*}$ Under Arbitrary Switching}

The subspace $\mathcal{V}_{R}^{*}$ is said to be outer quadratically stabilizable under arbitrary switching if there exists a friend $F_{\sigma_{t}}$ such that the induced switching dynamics $\left.\left(A_{\sigma_{t}}+B_{\sigma_{t}} F_{\sigma_{t}}\right)\right|_{\mathcal{X} / \mathcal{V}_{R}^{*}}$ is quadratically stable under arbitrary switching.

A convex procedure to compute, if possible, a friend $\left\{F_{\sigma}, \sigma \in \mathcal{I}\right\}$ such that $\mathcal{V}_{R}^{*}$ is a robust $\left(A_{\sigma}+B_{\sigma} F_{\sigma}\right)$-invariant subspace and is outer quadratically stable under arbitrary switching will be presented in Section 4, as part of the solution to the structural disturbance decoupling problem. However, at this stage, it is important to highlight possible degrees of freedom in the choice of $\left\{F_{\sigma}, \sigma \in \mathcal{I}\right\}$ or, conversely, to find obstructions to outer quadratic stabilization of $\mathcal{V}_{R}^{*}$.

The notion of reachable subspace, applied to each mode of the switching system, allows us to define a mode-dependent state space basis transformation such that, in the new coordinates, the assignable and the fixed outer dynamics of $\mathcal{V}_{R}^{*}$, with respect to the corresponding mode of the switching system, are neatly detached. Then, a necessary condition for the subspace $\mathcal{V}_{R}^{*}$ to be outer quadratically stabilizable under arbitrary switching is derived. 
Lemma 2. Consider the modes $\left\{\Sigma_{\sigma}, \sigma \in \mathcal{I}\right\}$, the subspace $\mathcal{V}_{R}^{*}$, and a friend $\left\{F_{\sigma}, \sigma \in \mathcal{I}\right\}$. For any $\sigma \in \mathcal{I}$, consider the reachable subspace $\mathcal{R}_{\sigma}$ of the pair $\left(A_{\sigma}, B_{\sigma}\right)$. Perform a state space basis transformation $T_{\sigma}=\left[\begin{array}{lll}T_{1} & T_{2, \sigma} & T_{3, \sigma}\end{array}\right]$, with $\operatorname{Im} T_{1}=\mathcal{V}_{R}^{*}$ and $\operatorname{Im}\left[T_{1} T_{2, \sigma}\right]=\mathcal{V}_{R}^{*}+\mathcal{R}_{\sigma}$. Also perform the control input space basis transformation $U_{\sigma}=\left[\begin{array}{lll}U_{1, \sigma} & U_{2, \sigma}\end{array}\right]$, with $\operatorname{Im} U_{1, \sigma}=B_{\sigma}^{-1} \mathcal{V}_{R}^{*}$. Then, for any $\sigma \in \mathcal{I}, A_{\sigma}^{\prime}+B_{\sigma}^{\prime} F_{\sigma}^{\prime}=T_{\sigma}^{-1}\left(A_{\sigma}+B_{\sigma} F_{\sigma}\right) T_{\sigma}, B_{\sigma}^{\prime}=T_{\sigma}^{-1} B_{\sigma} U_{\sigma}, H_{\sigma}^{\prime}=T_{\sigma}^{-1} H_{\sigma}$, and $C_{\sigma}^{\prime}=C_{\sigma} T_{\sigma}$ have the structure:

$$
\begin{aligned}
& A_{\sigma}^{\prime}+B_{\sigma}^{\prime} F_{\sigma}^{\prime}= \\
& {\left[\begin{array}{ccc}
A_{11, \sigma}^{\prime}+B_{11, \sigma}^{\prime} F_{11, \sigma}^{\prime}+ & A_{12, \sigma}^{\prime}+B_{11, \sigma}^{\prime} F_{12, \sigma}^{\prime}+ & A_{13, \sigma}^{\prime}+B_{11, \sigma}^{\prime} F_{13, \sigma}^{\prime}+ \\
B_{12, \sigma}^{\prime} F_{21, \sigma}^{\prime} & B_{12, \sigma}^{\prime} F_{22, \sigma}^{\prime} & B_{12, \sigma}^{\prime} F_{23, \sigma}^{\prime} \\
0 & A_{22, \sigma}^{\prime}+B_{22, \sigma}^{\prime} F_{22, \sigma}^{\prime} & A_{23, \sigma}^{\prime}+B_{22, \sigma}^{\prime} F_{23, \sigma}^{\prime} \\
0 & 0 & A_{33, \sigma}^{\prime}
\end{array}\right]} \\
& B_{\sigma}^{\prime}=\left[\begin{array}{cc}
B_{11, \sigma}^{\prime} & B_{12, \sigma}^{\prime} \\
0 & B_{22, \sigma}^{\prime} \\
0 & 0
\end{array}\right], \quad H_{\sigma}^{\prime}=\left[\begin{array}{c}
H_{1, \sigma}^{\prime} \\
H_{2, \sigma}^{\prime} \\
H_{3, \sigma}^{\prime}
\end{array}\right], \quad C_{\sigma}^{\prime}=\left[\begin{array}{lll}
0 & C_{2, \sigma}^{\prime} & C_{3, \sigma}^{\prime}
\end{array}\right] .
\end{aligned}
$$

Proof. The zero matrices in first block of columns of $A_{\sigma}^{\prime}+B_{\sigma}^{\prime} F_{\sigma}^{\prime}$ are due to $\mathcal{V}_{R}^{*}$ being an $\left(A_{\sigma}+B_{\sigma} F_{\sigma}\right)$-invariant subspace. The zero matrices in the last block of rows of $A_{\sigma}^{\prime}+B_{\sigma}^{\prime} F_{\sigma}^{\prime}$ are due to $\mathcal{V}_{R}^{*}+\mathcal{R}_{\sigma}$ being an $\left(A_{\sigma}+B_{\sigma} F_{\sigma}\right)$-invariant subspace. Indeed, $\mathcal{R}_{\sigma}$ is an $\left(A_{\sigma}+B_{\sigma} F_{\sigma}\right)$-invariant subspace because it is an $A_{\sigma}$-invariant subspace containing $\mathcal{B}_{\sigma}$. Moreover, the set of all $\left(A_{\sigma}+B_{\sigma} F_{\sigma}\right)$ invariant subspaces is closed with respect to the sum. The zero matrices in the first block of columns of $B_{\sigma}^{\prime}$ are due to $B_{\sigma}\left(\operatorname{Im} U_{1, \sigma}\right) \subseteq \mathcal{V}_{R}^{*}$. The zero matrices in the last block of rows of $B_{\sigma}^{\prime}$ are due to $\mathcal{B}_{\sigma} \subseteq \mathcal{R}_{\sigma} \subseteq \mathcal{V}_{R}^{*}+\mathcal{R}_{\sigma}$. The zero matrix in $C_{\sigma}^{\prime}$ is due to $\mathcal{V}_{R}^{*} \subseteq \mathcal{C} \subseteq \mathcal{C}_{\sigma}$.

It is worthwhile observing that the new state space basis transformation is congruent with that considered in Lemma 1, since it differs from the former only in the finer partition of the vectors that complete the basis of $\mathcal{V}_{R}^{*}$ to the state space $\mathcal{X}$. Therefore, for any $\sigma \in \mathcal{I}$, the outer dynamics of $\mathcal{V}_{R}^{*}$ with respect to the mode $\Sigma_{\sigma}$ - namely, the restriction $\left.\left(A_{\sigma}+B_{\sigma} F_{\sigma}\right)\right|_{\mathcal{X} / \mathcal{V}_{R}^{*}}$ - is represented by the 2-by-2-block matrix in the lower right corner of $A_{\sigma}^{\prime}+B_{\sigma}^{\prime} F_{\sigma}^{\prime}$. Moreover, the restriction $\left.\left(A_{\sigma}+B_{\sigma} F_{\sigma}\right)\right|_{\mathcal{X} /\left(\mathcal{V}_{R}^{*}+\mathcal{R}_{\sigma}\right)}$ is represented by the matrix in the lower right corner of $A_{\sigma}^{\prime}+B_{\sigma}^{\prime} F_{\sigma}^{\prime}$. Since the pair $\left(A_{22, \sigma}^{\prime}, B_{22, \sigma}^{\prime}\right)$ is controllable by construction, the eigenvalues of the restriction $\left.\left(A_{\sigma}+B_{\sigma} F_{\sigma}\right)\right|_{\left(\mathcal{V}_{R}^{*}+\mathcal{R}_{\sigma}\right) / \mathcal{V}_{R}^{*}}$ can be assigned through a suitable choice of $F_{22, \sigma}^{\prime}$. For this reason, the restriction $\left.\left(A_{\sigma}+B_{\sigma} F_{\sigma}\right)\right|_{\left(\mathcal{V}_{R}^{*}+\mathcal{R}_{\sigma}\right) / \mathcal{V}_{R}^{*}}$ is called the assignable outer dynamics of $\mathcal{V}_{R}^{*}$ with respect to $\Sigma_{\sigma}$. Instead, the eigenvalues of $A_{33, \sigma}^{\prime}$ are fixed and the restriction 
$\left.\left(A_{\sigma}+B_{\sigma} F_{\sigma}\right)\right|_{\mathcal{X} /\left(\mathcal{V}_{R}^{*}+\mathcal{R}_{\sigma}\right)}$ is called the unassignable outer dynamics of $\mathcal{V}_{R}^{*}$ with respect to $\Sigma_{\sigma}$. In light of these remarks, the following theorem gives a necessary condition for the subspace $\mathcal{V}_{R}^{*}$ to be outer quadratically stabilizable under arbitrary switching.

Theorem 1. The subspace $\mathcal{V}_{R}^{*}$ is outer quadratically stabilizable under arbitrary switching only if $\lambda\left(A_{33, \sigma}^{\prime}\right) \subset \mathbb{C}^{\odot}$ for all $\sigma \in \mathcal{I}$, with $A_{33, \sigma}^{\prime}$ defined as in Lemma 2.

Proof. Let $F_{\sigma_{t}}$ be a friend of $\mathcal{V}_{R}^{*}$. Thus, the outer switching dynamics of $\mathcal{V}_{R}^{*}$ is the switching dynamics $\left.\left(A_{\sigma_{t}}+B_{\sigma_{t}} F_{\sigma_{t}}\right)\right|_{\mathcal{X}} / \mathcal{V}_{R}^{*}$, associated to the set of restrictions $\left\{\left.\left(A_{\sigma}+B_{\sigma} F_{\sigma}\right)\right|_{\mathcal{X} / \mathcal{V}_{R}^{*}}, \sigma \in \mathcal{I}\right\}$. As is well known [38], a necessary condition for a switching dynamics to be quadratically stable under arbitrary switching is that all its modes are asymptotically stable. Hence, the outer switching dynamics of $\mathcal{V}_{R}^{*}$ is quadratically stable under arbitrary switching only if $\lambda\left(\left.\left(A_{\sigma}+B_{\sigma} F_{\sigma}\right)\right|_{\mathcal{X} / \mathcal{V}_{R}^{*}}\right) \subset \mathbb{C}^{\odot}$, for all $\sigma \in \mathcal{I}$. By virtue of Lemma 2, for any $\sigma \in \mathcal{I}$

$$
\begin{aligned}
& \lambda\left(\left(A_{\sigma}+B_{\sigma} F_{\sigma}\right) \mid \mathcal{X} \mathcal{V}_{R}^{*}\right) \\
& \quad=\lambda\left(\left.\left(A_{\sigma}+B_{\sigma} F_{\sigma}\right)\right|_{\left.\left(\mathcal{V}_{R}^{*}+\mathcal{R}_{\sigma}\right) / \mathcal{V}_{R}^{*}\right)} \uplus \lambda\left(\left.\left(A_{\sigma}+B_{\sigma} F_{\sigma}\right)\right|_{\mathcal{X} /\left(\mathcal{V}_{R}^{*}+\mathcal{R}_{\sigma}\right)}\right),\right.
\end{aligned}
$$

where $\lambda\left(\left.\left(A_{\sigma}+B_{\sigma} F_{\sigma}\right)\right|_{\left.\left(\mathcal{V}_{R}^{*}+\mathcal{R}_{\sigma}\right) / \mathcal{V}_{R}^{*}\right)}\right.$ is assignable, while

$$
\lambda\left(\left.\left(A_{\sigma}+B_{\sigma} F_{\sigma}\right)\right|_{\mathcal{X} /\left(\mathcal{V}_{R}^{*}+\mathcal{R}_{\sigma}\right)}\right)=\lambda\left(A_{33, \sigma}^{\prime}\right)
$$

is fixed.

\subsection{Inner Quadratic Stabilizability of $\mathcal{V}_{R}^{*}$ Under Arbitrary Switching}

The subspace $\mathcal{V}_{R}^{*}$ is said to be inner quadratically stabilizable under arbitrary switching if there exists a friend $F_{\sigma_{t}}$ such that the restricted switching dynamics $\left.\left(A_{\sigma_{t}}+B_{\sigma_{t}} F_{\sigma_{t}}\right)\right|_{\mathcal{V}_{R}^{*}}$ is quadratically stable under arbitrary switching.

Along the same lines followed in the discussion about outer stabilizability, the following arguments lead to a necessary condition for the subspace $\mathcal{V}_{R}^{*}$ to be inner quadratically stabilizable under arbitrary switching. To this aim, the reachability subspaces constrained to $\mathcal{V}_{R}^{*}$ of the respective modes of the switching system are introduced and, consequently, the assignable and the fixed inner dynamics of $\mathcal{V}_{R}^{*}$, with respect to each mode, are singled out. In the following, some basic notions of the geometric approach are extended to switching 
systems. To avoid heavy technicalities, some statements are presented without proof — the reader is referred to [2] for details on classic definitions and properties as well as for guidelines on omitted proofs.

The following statements lead to the definition of reachability subspace constrained to $\mathcal{V}_{R}^{*}$ for the generic mode $\Sigma_{\sigma}$ of the switching system $\Sigma_{\sigma_{t}}$. Beforehand, it is worth recalling that an $\left(A_{\sigma}, \mathcal{B}_{\sigma}\right)$-controlled invariant subspace $\mathcal{V}$ is said to be self-bounded with respect to $\mathcal{V}_{R}^{*}$ if $\mathcal{V} \subseteq \mathcal{V}_{R}^{*}$ and $\mathcal{V} \supseteq \mathcal{V}_{R}^{*} \cap \mathcal{B}_{\sigma}$. Moreover, self-bounded controlled invariant subspaces enjoy a couple of properties which are worth reviewing. First, if the linear map $F_{\sigma}$ is such that $\left(A_{\sigma}+B_{\sigma} F_{\sigma}\right) \mathcal{V}_{R}^{*} \subseteq \mathcal{V}_{R}^{*}$ holds, then $\left(A_{\sigma}+B_{\sigma} F_{\sigma}\right) \mathcal{V} \subseteq \mathcal{V}$ holds for any $\left(A_{\sigma}, \mathcal{B}_{\sigma}\right)$ controlled invariant subspace $\mathcal{V}$ self-bounded with respect to $\mathcal{V}_{R}^{*}$. Second, the intersection of two $\left(A_{\sigma}, \mathcal{B}_{\sigma}\right)$-controlled invariant subspaces self-bounded with respect to $\mathcal{V}_{R}^{*}$ is an $\left(A_{\sigma}, \mathcal{B}_{\sigma}\right)$-controlled invariant subspace self-bounded with respect to $\mathcal{V}_{R}^{*}$. In particular, the latter property implies that the set of all $\left(A_{\sigma}, \mathcal{B}_{\sigma}\right)$-controlled invariant subspaces self-bounded with respect to $\mathcal{V}_{R}^{*}$ is a lattice, with the sum and the intersection as binary operations and the inclusion as partial ordering relation. Thus, the constrained reachability subspace on $\mathcal{V}_{R}^{*}$ of the mode $\Sigma_{\sigma}$ - henceforth denoted by $\mathcal{R}_{\mathcal{V}_{R}^{*}, \sigma}$ - is defined as the minimum of the lattice of all $\left(A_{\sigma}, \mathcal{B}_{\sigma}\right)$-controlled invariant subspaces self-bounded with respect to $\mathcal{V}_{R}^{*}$.

Further, for any $\sigma \in \mathcal{I}$, the constrained reachability subspace $\mathcal{R}_{\mathcal{V}_{R}^{*}, \sigma}$ can be shown to be the intersection of $\mathcal{V}_{R}^{*}$ with a subspace introduced as follows. First, it is worth recalling that a subspace $\mathcal{S}$ is said to be an $\left(A_{\sigma}, \mathcal{V}_{R}^{*}\right)$-conditioned invariant subspace if $A_{\sigma}\left(\mathcal{S} \cap \mathcal{V}_{R}^{*}\right) \subseteq \mathcal{S}$. Moreover, simple algebraic arguments show that the set of all $\left(A_{\sigma}, \mathcal{V}_{R}^{*}\right)$-conditioned invariant subspaces containing $\mathcal{B}_{\sigma}$ is a lower semilattice with respect to the intersection and the inclusion of subspaces. The minimum of the semilattice is denoted by $\mathcal{S}_{\mathcal{V}_{R}^{*}, \sigma}$. Hence, it can be shown that $\mathcal{R}_{\mathcal{V}_{R}^{*}, \sigma}$ coincides with $\mathcal{V}_{R}^{*} \cap \mathcal{S}_{\mathcal{V}_{R}^{*}, \sigma}$. The subspaces $\mathcal{R}_{\mathcal{V}_{R}^{*}, \sigma}$ and $\mathcal{S}_{\mathcal{V}_{R}^{*}, \sigma}$ allow us to define a mode-dependent state space basis trasformation $T_{\sigma}$ such that, with respect to the new coordinates, the assignable and the fixed inner dynamics of the subspace $\mathcal{V}_{R}^{*}$, with respect to each mode $\Sigma_{\sigma}$, are separate.

Lemma 3. Consider the modes $\left\{\Sigma_{\sigma}, \sigma \in \mathcal{I}\right\}$, the subspace $\mathcal{V}_{R}^{*}$, and a friend $F_{\sigma_{t}}$. For any $\sigma \in \mathcal{I}$, consider the subspaces $\mathcal{S}_{\mathcal{V}_{R}^{*}, \sigma}$ and $\mathcal{R}_{\mathcal{V}_{R}^{*}, \sigma}$. Perform the state

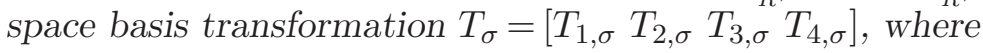

$$
\operatorname{Im} T_{1, \sigma}=\mathcal{R}_{\mathcal{V}_{R}^{*}, \sigma}, \quad \operatorname{Im}\left[T_{1, \sigma} T_{2, \sigma}\right]=\mathcal{V}_{R}^{*},
$$

and

$$
\operatorname{Im}\left[T_{1, \sigma} T_{3, \sigma}\right]=\mathcal{S}_{\mathcal{V}_{R}^{*}, \sigma}
$$


Also perform the control input space basis transformation $U_{\sigma}=\left[U_{1, \sigma} U_{2, \sigma}\right]$, where $\operatorname{Im} U_{1, \sigma}=B_{\sigma}^{-1} \mathcal{V}_{R}^{*}$. Then, for any $\sigma \in \mathcal{I}$,

$$
\begin{gathered}
A_{\sigma}^{\prime \prime}+B_{\sigma}^{\prime \prime} F_{\sigma}^{\prime \prime}=T_{\sigma}^{-1}\left(A_{\sigma}+B_{\sigma} F_{\sigma}\right) T_{\sigma}, \\
B_{\sigma}^{\prime \prime}=T_{\sigma}^{-1} B_{\sigma} U_{\sigma}, \\
H_{\sigma}^{\prime \prime}=T_{\sigma}^{-1} H_{\sigma},
\end{gathered}
$$

and

$$
C_{\sigma}^{\prime \prime}=C_{\sigma} T_{\sigma}
$$

have the structure:

$$
\begin{aligned}
& A_{\sigma}^{\prime \prime}+B_{\sigma}^{\prime \prime} F_{\sigma}^{\prime \prime}= \\
& {\left[\begin{array}{cccc}
A_{11, \sigma}^{\prime \prime}+B_{11, \sigma}^{\prime \prime} F_{11, \sigma}^{\prime \prime} & A_{12, \sigma}^{\prime \prime}+B_{11, \sigma}^{\prime \prime} F_{12, \sigma}^{\prime \prime} & A_{13, \sigma}^{\prime \prime}+B_{11, \sigma}^{\prime \prime} F_{13, \sigma}^{\prime \prime} & A_{14, \sigma}^{\prime \prime}+B_{11, \sigma}^{\prime \prime} F_{14, \sigma}^{\prime \prime} \\
+B_{12, \sigma}^{\prime \prime} F_{21, \sigma}^{\prime \prime} & +B_{12, \sigma}^{\prime \prime} F_{22, \sigma}^{\prime \prime} & +B_{12, \sigma}^{\prime \prime} F_{23, \sigma}^{\prime \prime} & +B_{12, \sigma}^{\prime \prime} F_{24, \sigma}^{\prime \prime} \\
0 & A_{22, \sigma}^{\prime \prime} & A_{23, \sigma}^{\prime \prime} & A_{24, \sigma}^{\prime \prime} \\
0 & 0 & A_{33, \sigma}^{\prime \prime}+B_{32, \sigma}^{\prime \prime} F_{23, \sigma}^{\prime \prime} & A_{34, \sigma}^{\prime \prime}+B_{32, \sigma}^{\prime \prime} F_{24, \sigma}^{\prime \prime} \\
0 & 0 & A_{43, \sigma}^{\prime \prime} & A_{44, \sigma}^{\prime \prime}
\end{array}\right]} \\
& B_{\sigma}^{\prime \prime}=\left[\begin{array}{cc}
B_{11, \sigma}^{\prime \prime} & B_{12, \sigma}^{\prime \prime} \\
0 & 0 \\
0 & B_{32, \sigma}^{\prime \prime} \\
0 & 0
\end{array}\right], \quad H_{\sigma}^{\prime \prime}=\left[\begin{array}{c}
H_{1, \sigma}^{\prime \prime} \\
H_{2, \sigma}^{\prime \prime} \\
H_{3, \sigma}^{\prime \prime} \\
H_{4, \sigma}^{\prime \prime}
\end{array}\right], \quad C_{\sigma}^{\prime \prime}=\left[\begin{array}{llll}
0 & 0 & C_{3, \sigma}^{\prime \prime} & C_{4, \sigma}^{\prime \prime}
\end{array}\right] .
\end{aligned}
$$

Proof. The zero matrices in the first block of columns of $A_{\sigma}^{\prime \prime}+B_{\sigma}^{\prime \prime} F_{\sigma}^{\prime \prime}$ are due to $\mathcal{R}_{\mathcal{V}_{R}^{*}, \sigma}$ being an $\left(A_{\sigma}+B_{\sigma} F_{\sigma}\right)$-invariant subspace. The zero matrices in the last two blocks of rows of $A_{\sigma}^{\prime \prime}+B_{\sigma}^{\prime \prime} F_{\sigma}^{\prime \prime}$ are due to $\mathcal{V}_{R}^{*}$ being an $\left(A_{\sigma}+B_{\sigma} F_{\sigma}\right)$ invariant subspace. The zero matrices in the first block of columns of $B_{\sigma}^{\prime \prime}$ are due to $B_{\sigma}\left(\operatorname{Im} U_{1, \sigma}\right) \subseteq \mathcal{V}_{R}^{*} \cap \mathcal{B}_{\sigma}=\mathcal{R}_{\mathcal{V}_{R}^{*}, \sigma} \cap \mathcal{B}_{\sigma}$. The zero matrices in the second and fourth blocks of rows of $B_{\sigma}^{\prime \prime}$ are due to $\mathcal{B}_{\sigma} \subseteq \mathcal{S}_{\mathcal{V}_{R}^{*}, \sigma}$. The zero matrices in $C_{\sigma}^{\prime \prime}$ are due to $\mathcal{V}_{R}^{*} \subseteq \mathcal{C} \subseteq \mathcal{C}_{\sigma}$.

It is worth noting that the state space basis transformation considered in Lemma 3 is consistent with that considered in Lemma 1, since it differs from that one in the more subtle, mode-dependent partition of the vectors of the basis of $\mathcal{V}_{R}^{*}$ as well as in the partition of the vectors that complete the basis of $\mathcal{V}_{R}^{*}$ to the state space $\mathcal{X}$. In view of this, the inner dynamics of $\mathcal{V}_{R}^{*}$ with respect to the mode $\Sigma_{\sigma}$ - i.e., the restriction $\left.\left(A_{\sigma}+B_{\sigma} F_{\sigma}\right)\right|_{\mathcal{V}_{R}^{*}} ^{*}$ - is represented by the 2-by-2-block matrix in the upper left corner of $A_{\sigma}^{\prime \prime}+B_{\sigma}^{\prime \prime} F_{\sigma}^{\prime \prime}$. Moreover, the inner dynamics of $\mathcal{R}_{\mathcal{V}_{R}^{*}, \sigma}$ with respect to $\Sigma_{\sigma}$ - i.e., the restriction $\left.\left(A_{\sigma}+B_{\sigma} F_{\sigma}\right)\right|_{\mathcal{R}_{\mathrm{V}_{R}^{*}, \sigma}}$ 
- is represented by the matrix in the upper left corner of $A_{\sigma}^{\prime \prime}+B_{\sigma}^{\prime \prime} F_{\sigma}^{\prime \prime}$. Since the pair $\left(A_{11, \sigma}^{\prime \prime}, B_{11, \sigma}^{\prime \prime}\right)$ is controllable by construction, the eigenvalues of the restriction $\left.\left(A_{\sigma}+B_{\sigma} F_{\sigma}\right)\right|_{\mathcal{R}_{\mathrm{V}_{R}^{*}, \sigma}}$ are assignable by a suitable choice of $F_{11, \sigma}^{\prime \prime}$. For this reason, $\left.\left(A_{\sigma}+B_{\sigma} F_{\sigma}\right)\right|_{\mathcal{R}_{\mathrm{V}_{R}^{*}, \sigma}}$ is called the assignable inner dynamics of $\mathcal{V}_{R}^{*}$ with respect to $\Sigma_{\sigma}$. On the contrary, the eigenvalues of $A_{22, \sigma}^{\prime \prime}$ are fixed and the restriction $\left.\left(A_{\sigma}+B_{\sigma} F_{\sigma}\right)\right|_{\mathcal{V}_{R}^{*} / \mathcal{R}_{\mathrm{V}_{R}^{*}, \sigma}}$ is called the unassignable inner dynamics of $\mathcal{V}_{R}^{*}$ with respect to $\Sigma_{\sigma}$. Based on these considerations, the following theorem provides a necessary condition for $\mathcal{V}_{R}^{*}$ to be inner quadratically stabilizable under arbitrary switching.

Theorem 2. The subspace $\mathcal{V}_{R}^{*}$ is inner quadratically stabilizable under arbitrary switching only if $\lambda\left(A_{22, \sigma}^{\prime \prime}\right) \subset \mathbb{C}^{\odot}$ for all $\sigma \in \mathcal{I}$, with $A_{22, \sigma}^{\prime \prime}$ defined as in Lemma 3.

Proof. Let $F_{\sigma_{t}}$ be a friend of $\mathcal{V}_{R}^{*}$. Thus, the inner switching dynamics of $\mathcal{V}_{R}^{*}$ is the switching dynamics $\left.\left(A_{\sigma_{t}}+B_{\sigma_{t}} F_{\sigma_{t}}\right)\right|_{\mathcal{V}_{R}^{*}}$, associated to the set of restrictions $\left\{\left.\left(A_{\sigma}+B_{\sigma} F_{\sigma}\right)\right|_{\mathcal{V}_{R}^{*}}, \sigma \in \mathcal{I}\right\}$. As was mentioned in the proof of Theorem 1, a switching dynamics is quadratically stable under arbitrary switching only if all the modes are asymptotically stable. Hence, the inner switching dynamics of $\mathcal{V}_{R}^{*}$ is quadratically stable under arbitrary switching only if $\lambda\left(\left.\left(A_{\sigma}+B_{\sigma} F_{\sigma}\right)\right|_{\mathcal{V}_{R}^{*}}\right) \subset \mathbb{C}^{\odot}$, for all $\sigma \in \mathcal{I}$. In light of Lemma 3, for any $\sigma \in \mathcal{I}$, $\lambda\left(\left.\left(A_{\sigma}+B_{\sigma} F_{\sigma}\right)\right|_{\mathcal{V}_{R}^{*}} ^{*}\right)=\lambda\left(\left.\left(A_{\sigma}+B_{\sigma} F_{\sigma}\right)\right|_{\mathcal{R}_{\mathrm{V}_{R}^{*}, \sigma}}\right) \uplus \lambda\left(\left.\left(A_{\sigma}+B_{\sigma} F_{\sigma}\right)\right|_{\mathcal{V}_{R}^{*} / \mathcal{R}_{\mathrm{V}_{R}^{*}, \sigma}}\right)$, where $\lambda\left(\left.\left(A_{\sigma}+B_{\sigma} F_{\sigma}\right)\right|_{\mathcal{R}_{\mathrm{V}_{R}^{*}, \sigma}}\right)$ is assignable, while $\lambda\left(\left.\left(A_{\sigma}+B_{\sigma} F_{\sigma}\right)\right|_{\mathcal{V}_{R}^{*} / \mathcal{R}_{\mathrm{V}_{R}^{*}, \sigma}}\right)=\lambda\left(A_{22, \sigma}^{\prime \prime}\right)$ is fixed.

By inspecting the partition, shown in (1), of the matrices representing the linear maps of the set $\left\{A_{\sigma}+B_{\sigma} F_{\sigma}, \sigma \in \mathcal{I}\right\}$ with respect to the coordinates of Lemma 1, one can see that the properties of inner and outer quadratic stabilizability of $\mathcal{V}_{R}^{*}$ are independent of each other.

\section{Structural Disturbance Decoupling}

This section deals with structural disturbance decoupling — namely, the problem of disturbance decoupling where the only concern is making the output insensitive to the disturbance, without caring about stability. In the following, the disturbance is assumed to be measurable. Then, it will be shown how the compensation scheme is modified when the disturbance is inaccessible. 
Problem 1 (Structural Disturbance Decoupling). Given the discrete-time switching linear system $\Sigma_{\sigma_{t}}$, find a switching control law

$$
u_{t}=F_{\sigma_{t}} x_{t}+G_{\sigma_{t}} h_{t}, \quad t \in \mathbb{Z}^{+},
$$

such that the compensated discrete-time switching linear system

$$
\hat{\Sigma}_{\sigma_{t}} \equiv\left\{\begin{aligned}
x_{t+1} & =\left(A_{\sigma_{t}}+B_{\sigma_{t}} F_{\sigma_{t}}\right) x_{t}+\left(H_{\sigma_{t}}+B_{\sigma_{t}} G_{\sigma_{t}}\right) h_{t}, \\
y_{t} & =C_{\sigma_{t}} x_{t},
\end{aligned}\right.
$$

satisfies the following requirement:

$\mathcal{R} 1$. the output $y_{t} \rightarrow 0$ as $t \rightarrow \infty$, for any admissible measurable disturbance $h_{t}$, with $t \in \mathbb{Z}^{+}$, any initial state $x_{0} \in \mathbb{R}^{n}$, and any switching signal $\sigma_{t}$.

The main theorem of this section will provide a pair of sufficient conditions for Problem 1 to be solvable. The proof is constructive, since it shows how to synthesize the switching control law. Arguments showing why the pair of conditions is not necessary are given. Two lemmas are premised, respectively expressing the two conditions, which are initially presented in coordinate-free terms, with respect to the coordinates introduced in Lemma 1.

Lemma 4. Consider the modes $\left\{\Sigma_{\sigma}, \sigma \in \mathcal{I}\right\}$, the subspace $\mathcal{V}_{R}^{*}$, and the subspaces $\mathcal{B}_{\sigma}$ and $\mathcal{H}_{\sigma}$, with $\sigma \in \mathcal{I}$. Let the subspaces $\mathcal{V}_{\sigma} \subseteq \mathcal{V}_{R}^{*}$, with $\sigma \in \mathcal{I}$, be such that $\mathcal{V}_{\sigma} \cap \mathcal{B}_{\sigma}=\{0\}$ and $\left(\mathcal{V}_{R}^{*} \cap \mathcal{B}_{\sigma}\right)+\mathcal{V}_{\sigma}=\mathcal{V}_{R}^{*}$. Refer to the coordinates introduced in Lemma 1 and let $V_{\sigma}^{*} \in \mathbb{R}^{n \times r_{\sigma}}$, with $\sigma \in \mathcal{I}$, be basis matrices of the corresponding subspaces $\mathcal{V}_{\sigma}$. Then,

$$
\mathcal{H}_{\sigma} \subseteq \mathcal{B}_{\sigma}+\mathcal{V}_{R}^{*}, \quad \text { for all } \sigma \in \mathcal{I},
$$

if and only if there exist $\Gamma_{\sigma} \in \mathbb{R}^{p \times m}$ and $\Lambda_{\sigma} \in \mathbb{R}^{r_{\sigma} \times m}$, with $\sigma \in \mathcal{I}$, such that

$$
H_{\sigma}^{*}=B_{\sigma}^{*} \Gamma_{\sigma}+V_{\sigma}^{*} \Lambda_{\sigma}, \quad \text { for all } \sigma \in \mathcal{I},
$$

or, with respect to the partition introduced in Lemma 1,

$$
\left[\begin{array}{l}
H_{1, \sigma}^{*} \\
H_{2, \sigma}^{*}
\end{array}\right]=\left[\begin{array}{c}
B_{1, \sigma}^{*} \\
B_{2, \sigma}^{*}
\end{array}\right] \Gamma_{\sigma}+\left[\begin{array}{c}
V_{1, \sigma}^{*} \\
0
\end{array}\right] \Lambda_{\sigma}, \quad \text { for all } \sigma \in \mathcal{I} .
$$

Proof. First, note that $\left(\mathcal{V}_{R}^{*} \cap \mathcal{B}_{\sigma}\right)+\mathcal{V}_{\sigma}=\mathcal{V}_{R}^{*}$ is equivalent to

$$
\mathcal{B}_{\sigma}+\mathcal{V}_{\sigma}=\mathcal{B}_{\sigma}+\mathcal{V}_{R}^{*}
$$

with $\sigma \in \mathcal{I}$, since $\mathcal{V}_{\sigma} \subseteq \mathcal{V}_{R}^{*}$ and $\mathcal{V}_{\sigma} \cap \mathcal{B}_{\sigma}=\{0\}$. Hence, (5) holds if and only if $\mathcal{H}_{\sigma} \subseteq \mathcal{B}_{\sigma}+\mathcal{V}_{\sigma}$ for all $\sigma \in \mathcal{I}$. Equivalently, any basis matrix of $\mathcal{H}_{\sigma}$ can be 
written as a linear combination of the column vectors of basis matrices of $\mathcal{B}_{\sigma}$ and $\mathcal{V}_{\sigma}$, for all $\sigma \in \mathcal{I}$. Since $B_{\sigma}$ and $H_{\sigma}$ are full-rank matrices for all $\sigma \in \mathcal{I}$ by assumption, so are $B_{\sigma}^{*}$ and $H_{\sigma}^{*}$, which refer to the coordinates introduced in Lemma 1. Hence, matrices $\Gamma_{\sigma} \in \mathbb{R}^{p \times m}$ and $\Lambda_{\sigma} \in \mathbb{R}^{r_{\sigma} \times m}$ exist, such that (6) holds. Moreover, $\mathcal{V}_{\sigma} \subseteq \mathcal{V}_{R}^{*}$ implies that $V_{\sigma}^{*}$ has a zero matrix in the last block of rows, as shown in (7).

Lemma 5. Consider the discrete-time switching linear system $\Sigma_{\sigma_{t}}$ and the subspace $\mathcal{V}_{R}^{*}$. Let $r=\operatorname{dim} \mathcal{V}_{R}^{*}$. The subspace $\mathcal{V}_{R}^{*}$ is outer quadratically stabilizable under arbitrary switching if and only if there exist a positive-definite symmetric matrix $Q_{e} \in \mathbb{R}^{(n-r) \times(n-r)}$ and a set of matrices $\left\{Y_{\sigma} \in \mathbb{R}^{p \times(n-r)}, \sigma \in \mathcal{I}\right\}$ such that

$$
\left[\begin{array}{cc}
Q_{e} & \left(A_{22, \sigma}^{*} Q_{e}+B_{2, \sigma}^{*} Y_{\sigma}\right)^{\top} \\
A_{22, \sigma}^{*} Q_{e}+B_{2, \sigma}^{*} Y_{\sigma} & Q_{e}
\end{array}\right]>0, \quad \text { for all } \sigma \in \mathcal{I},
$$

with $A_{22, \sigma}^{*}$ and $B_{2, \sigma}^{*}$ defined as in Lemma 1. If this is the case, the switching dynamics $\left.\left(A_{\sigma_{t}}+B_{\sigma_{t}} F_{\sigma_{t}}\right)\right|_{\mathcal{X} / \mathcal{V}_{R}^{*}}$, associated to the set of matrices

$$
\left\{A_{22, \sigma}^{*}+B_{2, \sigma}^{*} F_{2, \sigma}^{*}, \sigma \in \mathcal{I}\right\},
$$

where $F_{2, \sigma}^{*}=Y_{\sigma} Q_{e}^{-1}$, with $\sigma \in \mathcal{I}$, is quadratically stable under arbitrary switching.

Proof. In light of Lemma $1, \mathcal{V}_{R}^{*}$ being a robust $\left(A_{\sigma}, \mathcal{B}_{\sigma}\right)$-controlled invariant subspace, with the property of outer quadratic stabilizability under arbitrary switching, means that there exist a positive-definite symmetric matrix $P_{e} \in \mathbb{R}^{(n-r) \times(n-r)}$ and a set of matrices $\left\{F_{2, \sigma}^{*} \in \mathbb{R}^{p \times n-r}, \sigma \in \mathcal{I}\right\}$ such that

$$
\left(A_{22, \sigma}^{*}+B_{2, \sigma}^{*} F_{2, \sigma}^{*}\right)^{\top} P_{e}\left(A_{22, \sigma}^{*}+B_{2, \sigma}^{*} F_{2, \sigma}^{*}\right)-P_{e}<0 \text {, for all } \sigma \in \mathcal{I} \text {. }
$$

Then, (9) is shown to be equivalent to (8) by the change of variables $Q_{e}=P_{e}^{-1}$, $Y_{\sigma}=F_{2, \sigma}^{*} Q_{e}$, with $\sigma \in \mathcal{I}$, and the Schur complement.

Note that (9) is not jointly convex in the unknowns $P_{e}$ and $F_{2, \sigma}^{*}$, with $\sigma \in \mathcal{I}$, while (8) is an LMI in the unknowns $Q_{e}$ and $Y_{\sigma}$, with $\sigma \in \mathcal{I}$. Hence, Lemma 5 points out a convex procedure to derive (if the LMI problem is feasible) a set of matrices $\left\{F_{2, \sigma}^{*}, \sigma \in \mathcal{I}\right\}$. Furthermore, a set of matrices $\left\{F_{1, \sigma}^{*}, \sigma \in \mathcal{I}\right\}$ such that

$$
A_{21, \sigma}^{*}+B_{2, \sigma}^{*} F_{1, \sigma}^{*}=0, \quad \text { for all } \sigma \in \mathcal{I},
$$

always exists, owing to the equivalence between robust controlled invariance and robust invariance by state feedback mentioned in Section 2. 
Theorem 3. Consider the discrete-time switching linear system $\Sigma_{\sigma_{t}}$. Problem 1 has a solution if the following conditions hold:

C 1. $\mathcal{H}_{\sigma} \subseteq \mathcal{B}_{\sigma}+\mathcal{V}_{R}^{*}$ for all $\sigma \in \mathcal{I}$;

$\mathcal{C} 2 . \mathcal{V}_{R}^{*}$ is outer quadratically stabilizable under arbitrary switching.

Proof. Let Conditions $\mathcal{C} 1$ and $\mathcal{C} 2$ hold. Consider the switching control law (4). Let $G_{\sigma_{t}}$ be the switching linear map associated to $\left\{G_{\sigma}, \sigma \in \mathcal{I}\right\}$, defined by

$$
G_{\sigma}=-\Gamma_{\sigma}, \quad \text { for all } \sigma \in \mathcal{I},
$$

where the matrices $\Gamma_{\sigma}$, with $\sigma \in \mathcal{I}$, are given by Lemma 4 (which is possible by virtue of Condition $\mathcal{C} 1$ ). Moreover, let $F_{\sigma_{t}}$ be the switching linear map associated to a set $\left\{F_{\sigma}, \sigma \in \mathcal{I}\right\}$ determined according to Lemmas 1 and 5 (which is feasible owing to Condition $\mathcal{C} 2$ ). Then, it will be shown that the switching control law (4), where $F_{\sigma_{t}}$ and $G_{\sigma_{t}}$ are picked as specified above, solves Problem 1. First, note that, with respect to the coordinates introduced in Lemma 1, the modes $\left\{\hat{\Sigma}_{\sigma}, \sigma \in \mathcal{I}\right\}$ of the switching compensated system $\hat{\Sigma}_{\sigma_{t}}$ are described by

$\hat{\Sigma}_{\sigma} \equiv\left\{\begin{aligned} x_{1, t+1} & =\left(A_{11, \sigma}^{*}+B_{1, \sigma}^{*} F_{1, \sigma}^{*}\right) x_{1, t}+V_{1, \sigma}^{*} \Lambda_{\sigma} h_{t}+\left(A_{12, \sigma}^{*}+B_{1, \sigma}^{*} F_{2, \sigma}^{*}\right) x_{2, t}, \\ x_{2, t+1} & =\left(A_{22, \sigma}^{*}+B_{2, \sigma}^{*} F_{2, \sigma}^{*}\right) x_{2, t}, \\ y_{t} & =C_{2, \sigma}^{*} x_{2, t},\end{aligned}\right.$

where (7), (10), and (11) have been taken into account. Equation (12) shows that quadratic stability under arbitrary switching of the dynamics

$$
\left.\left(A_{\sigma_{t}}+B_{\sigma_{t}} F_{\sigma_{t}}\right)\right|_{\mathcal{X} / \mathcal{V}_{R}^{*}},
$$

associated to the set $\left\{A_{22, \sigma}^{*}+B_{2, \sigma}^{*} F_{2, \sigma}^{*}, \sigma \in \mathcal{I}\right\}$, implies that the state trajectory $x_{2, t}$, with $t \in \mathbb{Z}^{+}$, converges to 0 as $t$ goes to $\infty$, for any admissible disturbance $h_{t}$, with $t \in \mathbb{Z}^{+}$(indeed, $h_{t}$ does not appear in the equation of $x_{2, t}$ ), any initial state $x_{0} \in \mathbb{R}^{n}$, and any switching signal $\sigma_{t}$. Therefore, the same is true for the output $y_{t}$, with $t \in \mathbb{Z}^{+}$, which only depends on $x_{2, t}$.

Condition $\mathcal{C} 1$ is also necessary to solve Problem 1 . In fact, if Condition $\mathcal{C} 1$ is not met, no other robust $\left(A_{\sigma}, \mathcal{B}_{\sigma}\right)$-controlled invariant subspace contained in $\mathcal{C}$, say $\mathcal{V}_{R}$, exists, such that $\mathcal{H}_{\sigma} \subseteq \mathcal{B}_{\sigma}+\mathcal{V}_{R}$, for all $\sigma \in \mathcal{I}$, because the set of all robust $\left(A_{\sigma}, \mathcal{B}_{\sigma}\right)$-controlled invariant subspaces contained in $\mathcal{C}$ is an upper semilattice and $\mathcal{V}_{R}^{*}$ is its maximum.

Condition $\mathcal{C} 2$ is not necessary to solve Problem 1. In fact, a less conservative form of stability for the switching dynamics $\left.\left(A_{\sigma_{t}}+B_{\sigma_{t}} F_{\sigma_{t}}\right)\right|_{\mathcal{X} / \mathcal{V}_{R}^{*}}$, such 
as asymptotic stability under arbitrary switching, would be sufficient to guarantee convergence to zero of the output as the time goes to infinity. However, as mentioned earlier, focusing on quadratic stability under arbitrary switching has the advantage of ensuring local input-to-state stability.

Concerning the convex procedure for determining the switching state feedback, the degrees of freedom available in the determination of $\left\{F_{2, \sigma}^{*}, \sigma \in \mathcal{I}\right\}$ pointed out in Section 3.A are implicitly exploited in the solution of the LMIs of Lemma 5.

This section is concluded by the following two remarks, which point out that inaccessible disturbance decoupling and perfect disturbance decoupling can be regarded as respective special cases of Problem 1.

Remark 1. If the more restrictive condition

$\mathcal{C} 1^{\prime} . \mathcal{H}_{\sigma} \subseteq \mathcal{V}_{R}^{*}$, for all $\sigma \in \mathcal{I}$

holds in place of Condition $\mathcal{C} 1$, the feedforward action applied through $G_{\sigma_{t}}$ is not required anymore. Thus, the disturbance input $h_{t}$, with $t \in \mathbb{Z}^{+}$, is no longer needed to be measurable. In fact, if Condition $\mathcal{C} 1^{\prime}$ holds, Lemma 4 can be modified by replacing (6) with $H_{\sigma}^{*}=T_{1}^{*} \Lambda_{\sigma}$, for all $\sigma \in \mathcal{I}$, where $T_{1}^{*}=\left[\begin{array}{ll}I & 0\end{array}\right]^{\top}$ is a basis matrix of $\mathcal{V}_{R}^{*}$ with respect to the coordinates introduced in Lemma 1. Consequently, the control law (4) reduces to $u_{t}=F_{\sigma_{t}} x_{t}$, with $t \in \mathbb{Z}^{+}$, where $F_{\sigma_{t}}$ is still determined according to Lemmas 1 and 5. Namely, the switching state feedback alone achieves asymptotic decoupling of any admissible inaccessible disturbance, for any initial state $x_{0} \in \mathbb{R}^{n}$ and any switching signal $\sigma_{t}$.

Remark 2. If the initial state $x_{0}$ belongs to the subspace $\mathcal{V}_{R}^{*}$, then the output $y_{t}$ is zero for all $t \in \mathbb{Z}^{+}$, for any admissible measurable (or, inaccessible if Condition $\mathcal{C} 1^{\prime}$ holds) disturbance $h_{t}$, with $t \in \mathbb{Z}^{+}$, and any switching signal $\sigma_{t}$. In fact, in light of $(12)$, if $x_{0} \in \mathcal{V}_{R}^{*}$, the sole state component $x_{1,0}$ may be different from zero. Therefore, $x_{2,0}=0$ implies $x_{2, t}=0$ for all $t \in \mathbb{Z}^{+}$, which, in turn, implies $y_{t}=0$ for all $t \in \mathbb{Z}^{+}$. Namely, when $x_{0} \in \mathcal{V}_{R}^{*}$, perfect decoupling of any admissible measurable (or, respectively, inaccessible) disturbance is achieved for any switching signal $\sigma_{t}$.

\section{Disturbance Decoupling with Quadratic Stability Under Arbitrary Switching}

This section concerns an improved version of the disturbance decoupling problem, where the dynamics of the compensated system is required to be quadrat- 
ically stable under arbitrary switching. It will be shown that the new requirement is compatible with the existing one. A new convex procedure, independent of the one discussed in Section 4 and to be applied besides that in the synthesis of the control law, will be illustrated.

Problem 2 (Disturbance Decoupling with Quadratic Stability). Given the discrete-time switching linear system $\Sigma_{\sigma_{t}}$, find a switching control law (4), such that the compensated system $\hat{\Sigma}_{\sigma_{t}}$ satisfies Requirements $\mathcal{R} 1$ and

$\mathcal{R} 2$. the switching dynamics $A_{\sigma_{t}}+B_{\sigma_{t}} F_{\sigma_{t}}$ is quadratically stable under arbitrary switching.

Referring to the partition considered in Lemma 1, the next lemma shows how a set of matrices $\left\{F_{1, \sigma}^{*}, \sigma \in \mathcal{I}\right\}$ such that $\mathcal{V}_{R}^{*}$ is a robust $\left(A_{\sigma}+B_{\sigma} F_{\sigma}\right)$ invariant subspace, with the property of being inner quadratically stable under arbitrary switching, can be obtained as a solution, if it exists, of a convex problem with a linear constraint.

Lemma 6. The subspace $\mathcal{V}_{R}^{*}$ is inner quadratically stabilizable under arbitrary switching if and only if there exist a positive-definite symmetric matrix $Q_{i} \in \mathbb{R}^{r \times r}$, where $r=\operatorname{dim} \mathcal{V}_{R}^{*}$, and a set of matrices $\left\{W_{\sigma} \in \mathbb{R}^{p \times r}, \sigma \in \mathcal{I}\right\}$ such that

$$
\left\{\begin{array}{cc}
Q_{i} & \left(A_{11, \sigma}^{*} Q_{i}+B_{1, \sigma}^{*} W_{\sigma}\right)^{\top} \\
A_{11, \sigma}^{*} Q_{i}+B_{1, \sigma}^{*} W_{\sigma} & Q_{i}
\end{array}\right]>0, \quad \text { for all } \sigma \in \mathcal{I},
$$

with $A_{11, \sigma}^{*}, A_{21, \sigma}^{*}, B_{1, \sigma}^{*}, B_{2, \sigma}^{*}$ defined as in Lemma 1. If this is the case, the switching dynamics $\left.\left(A_{\sigma_{t}}+B_{\sigma_{t}} F_{\sigma_{t}}\right)\right|_{\mathcal{V}_{R}^{*}}$, associated to the set of matrices $\left\{A_{11, \sigma}^{*}+B_{1, \sigma}^{*} F_{1, \sigma}^{*}, \sigma \in \mathcal{I}\right\}$, where $F_{1, \sigma}^{*}=W_{\sigma} Q_{i}^{-1}$, with $\sigma \in \mathcal{I}$, is quadratically stable under arbitrary switching.

Proof. In light of Lemma $1, \mathcal{V}_{R}^{*}$ being a robust $\left(A_{\sigma}, \mathcal{B}_{\sigma}\right)$-controlled invariant subspace, with the property of inner quadratic stabilizability under arbitrary switching, means that there exist a positive-definite symmetric matrix $P_{i} \in \mathbb{R}^{r \times r}$ and a set of matrices $\left\{F_{1, \sigma}^{*} \in \mathbb{R}^{p \times r}, \sigma \in \mathcal{I}\right\}$ such that

$$
\left\{\begin{array}{l}
\left(A_{11, \sigma}^{*}+B_{1, \sigma}^{*} F_{1, \sigma}^{*}\right)^{\top} P_{i}\left(A_{11, \sigma}^{*}+B_{1, \sigma}^{*} F_{1, \sigma}^{*}\right)-P_{i}<0, \quad \text { for all } \sigma \in \mathcal{I} . \\
A_{21, \sigma}^{*}+B_{2, \sigma}^{*} F_{1, \sigma}^{*}=0,
\end{array}\right.
$$

Then, the first of (14) is shown to be equivalent to the first of (13) by the change of variables $Q_{i}=P_{i}^{-1}, W_{\sigma}=F_{1, \sigma}^{*} Q_{i}$, with $\sigma \in \mathcal{I}$, and the Schur complement. Further, the latter of (14) is proved to be equivalent to the latter of (13) by multiplying by $Q_{i}$ and applying the same change of variables. 
Theorem 4. Consider the discrete-time switching linear system $\Sigma_{\sigma_{t}}$. Problem 2 has a solution if Conditions $\mathcal{C} 1, \mathcal{C} 2$, and the following hold:

$\mathcal{C} 3 . \mathcal{V}_{R}^{*}$ is inner quadratically stabilizable under arbitrary switching.

Proof. Let Conditions $\mathcal{C} 1-\mathcal{C} 3$ hold. Consider the switching control law (4). Let $G_{\sigma_{t}}$ be determined as in the proof of Theorem 3. Let $F_{\sigma_{t}}$ be such that, with respect to the partition of the matrices $F_{\sigma}^{*}$, with $\sigma \in \mathcal{I}$, considered in Lemma 1 , the matrices $F_{2, \sigma}^{*}$, with $\sigma \in \mathcal{I}$, are derived according to Lemma 5 , as in the proof of Theorem 3, while the matrices $F_{1, \sigma}^{*}$, with $\sigma \in \mathcal{I}$, are derived according to Lemma 6 (which is feasible owing to Condition $\mathcal{C} 3$ ). Hence, the proof that Requirement $\mathcal{R} 1$ is satisfied is the same as in Theorem 3. As to Requirement $\mathcal{R} 2$, the proof follows from the upper block-triangular structure of the dynamics of $\Sigma_{\sigma_{t}}$ shown in (12) and from quadratic stability under arbitrary switching of the switching dynamics $\left.\left(A_{\sigma_{t}}+B_{\sigma_{t}} F_{\sigma_{t}}\right)\right|_{\mathcal{V}_{R}^{*}}$ and $\left.\left(A_{\sigma_{t}}+B_{\sigma_{t}} F_{\sigma_{t}}\right)\right|_{\mathcal{X} / \mathcal{V}_{R}^{*}}$ (see, e.g., [39, Chapter 7]).

To summarize the reasoning developed so far, structural disturbance decoupling has been tackled first. In the solution of that problem, only outer stabilization of the subspace $\mathcal{V}_{R}^{*}$ has been required, so as to guarantee asymptotic convergence of the output to zero in the presence of nonzero initial state. Then, disturbance decoupling with state stability has been solved, which has required stabilization of both inner and outer switching dynamics of $\mathcal{V}_{R}^{*}$, so as to guarantee state stability of the closed-loop switching system. In particular, quadratic stability under arbitrary switching has been required. Indeed, the existence of a common quadratic Lyapunov function is a sufficient condition for global uniform asymptotic stability of a switching system [38]. Moreover, zeroinput asymptotic stability of a nonlinear system implies local input-to-state stability [36] — this fact was considered with specific reference to switching systems in [34, Appendix A]. In light of these results, inner and outer quadratic stabilization of the subspace $\mathcal{V}_{R}^{*}$ under arbitrary switching guarantees not only disturbance decoupling with global uniform asymptotic stability of the switching system under zero input, but also input-to-state stability. 


\section{An Illustrative Example}

Let Problem 2 be stated for the switching linear system $\Sigma_{\sigma_{t}}$, defined by the index set $\mathcal{I}=\{1,2\}$ and the matrices

$$
\begin{aligned}
& A_{1}=\left[\begin{array}{cccccc}
0.6 & 0 & 0 & 0 & -0.3 & 0 \\
1 & 1 & -0.3 & 0 & 0 & 0 \\
0 & 0.4 & 0.85 & 0 & 0 & 0 \\
0 & 0 & 0 & 0.95 & 0 & -0.4 \\
0 & 0 & 0 & 0 & 0.5 & 0 \\
0 & 0 & 0 & 0 & 0 & 0.7
\end{array}\right], \quad B_{1}=\left[\begin{array}{lll}
1 & 0 & 1 \\
0 & 1 & 0 \\
2 & 0 & 1 \\
0 & 2 & 0 \\
1 & 0 & 0 \\
0 & 1 & 0
\end{array}\right], \\
& H_{1}=\left[\begin{array}{ccc}
-0.5 & 0 & 0 \\
0 & 0 & 0 \\
0 & 0 & 1 \\
0 & 0 & 0 \\
0.5 & 0 & 0 \\
0 & 1 & 0
\end{array}\right] \\
& A_{2}=\left[\begin{array}{cccccc}
0.4 & -0.1 & 0.1 & 0 & 0 & 0 \\
0.9 & 1 & -0.3 & 0 & 0 & 0 \\
0.6 & 0.4 & 0.2 & 0 & 0 & 0 \\
-1.2 & -0.3 & 0.1 & 0.5 & 0.1 & 0 \\
0.9 & 0.1 & 0.1 & 0 & 0.2 & 0.1 \\
-0.1 & 0 & 0 & 0 & 0 & 0.7
\end{array}\right], \quad B_{2}=\left[\begin{array}{lll}
1 & 1 & 0 \\
0 & 0 & 0 \\
0 & 0 & 1 \\
0 & 1 & 0 \\
1 & 0 & 0 \\
0 & 1 & 1
\end{array}\right], \\
& H_{2}=\left[\begin{array}{ccc}
0 & -1 & 0.5 \\
0 & -1 & 0 \\
0 & 0 & 0 \\
1 & 0 & 0 \\
0 & 0 & 0.5 \\
-3 & 0 & 0
\end{array}\right] \\
& C_{1}=\left[\begin{array}{llllll}
0 & 0 & 2 & 1 & 0 & 0 \\
0 & 0 & 0 & 1 & 0 & 0
\end{array}\right] \quad C_{2}=\left[\begin{array}{llllll}
0 & 0 & 2 & 0 & 0 & 0 \\
0 & 0 & 0 & 1 & 0 & 0
\end{array}\right]
\end{aligned}
$$

Let the sampling time be $T_{s}=0.1 \mathrm{~s}$. According to the proposed procedure,

$$
\mathcal{V}_{R}^{*}=\operatorname{Im}\left[\begin{array}{cccc}
1 & 0 & 0 & 0 \\
0 & -1 & 0 & 0 \\
0 & 0 & 0 & 0 \\
0 & 0 & 0 & 0 \\
0 & 0 & -1 & 0 \\
0 & 0 & 0 & 1
\end{array}\right] .
$$


Condition $\mathcal{C} 1$ of Theorem 3 is satisfied, as can be checked by comparing the basis matrices of the subspaces $\mathcal{H}_{\sigma}, \mathcal{B}_{\sigma}$, and $\mathcal{V}_{R}^{*}$ for all $\sigma \in \mathcal{I}$. Hence, the matrices $\Gamma_{\sigma}$ and $\Lambda_{\sigma}$, with $\sigma \in \mathcal{I}$, can be computed according to Lemma 4. Thus, the matrices of the set $\left\{G_{\sigma}, \sigma \in \mathcal{I}\right\}$, defined according to (11), are

$$
G_{1}=\left[\begin{array}{ccc}
-0.5 & 0 & -0.5 \\
0 & 0 & 0 \\
1 & 0 & 0
\end{array}\right], \quad G_{2}=\left[\begin{array}{ccc}
0.5 & 0.5 & -0.5 \\
-1 & 0 & 0 \\
0 & 0 & 0
\end{array}\right]
$$

The set $\left\{F_{\sigma}, \sigma \in \mathcal{I}\right\}$ is derived according to Lemmas 1,5 , and 6 . The solution to the LMI problem (8) and that to the LMI problem with linear constraint (13) show that Condition $\mathcal{C} 2$ of Theorem 3 and Condition $\mathcal{C} 3$ of Theorem 4 are both satisfied. Thus, a set of matrices $\left\{F_{\sigma}, \sigma \in \mathcal{I}\right\}$ that solves both the LMI problems is

$$
\begin{aligned}
& F_{1}=\left[\begin{array}{cccccc}
0.7526 & -0.0635 & 0 & 0 & -0.2974 & 0 \\
0 & 0 & 0 & 0 & 0 & 0.2 \\
-1.5052 & -0.2729 & 0 & 0 & 0.5948 & 0
\end{array}\right] \\
& F_{2}=\left[\begin{array}{cccccc}
-2.1725 & -1.0251 & 0 & 0 & 0.0725 & 0.1040 \\
1.2 & 0.3 & 0 & 0 & -0.1 & 0 \\
-0.6 & -0.4 & 0 & 0 & 0 & 0
\end{array}\right] .
\end{aligned}
$$

A simulation has been run to check the performance of the devised control law. The simulation time goes from $0 \mathrm{~s}$ to $10 \mathrm{~s}$ (the number of samples is 100). The switching signal $\sigma_{t}$ is equal to 1 , with $t=0, \ldots, 19$, equal to 2 , with $t=20, \ldots, 29$, and equal to 1 , with $t=30, \ldots, 99$ - i.e., the active mode is $\Sigma_{1}$, between $0 \mathrm{~s}$ and $1.9 \mathrm{~s}$ as well as between $3 \mathrm{~s}$ and $9.9 \mathrm{~s}$, while it is $\Sigma_{2}$ between $2 \mathrm{~s}$ and $2.9 \mathrm{~s}$. The components of the measurable disturbance respectively are a negative step with amplitude equal to 0.5 applied at the time $1 \mathrm{~s}$, a sinewave with amplitude equal to 2 and a sawtooth with slope equal to 0.5 and period equal to $2 \mathrm{~s}$, both applied at the time $0 \mathrm{~s}$. The initial state is $x_{0}=\left[\begin{array}{llllll}1 & -1 & 0.5 & 1 & -1 & 1\end{array}\right]^{\top}$. The output $y$ of the compensated system $\hat{\Sigma}_{\sigma_{t}}$ is shown in Fig. 1. Both components converge to zero, which shows that structural decoupling is achieved. The state trajectories are shown in Fig. 2. Note that they are bounded (in the presence of the bounded inputs). 


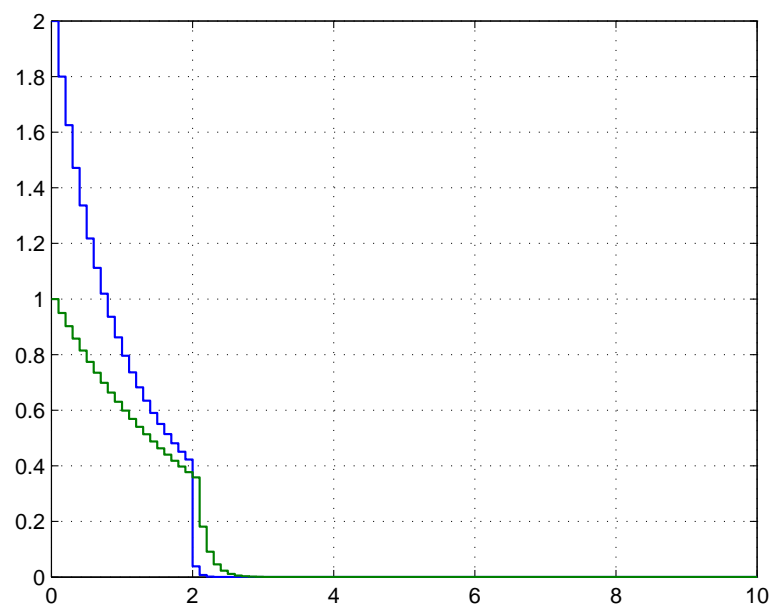

Figure 1: Output $y$ of the compensated system - amplitude vs. time (s)

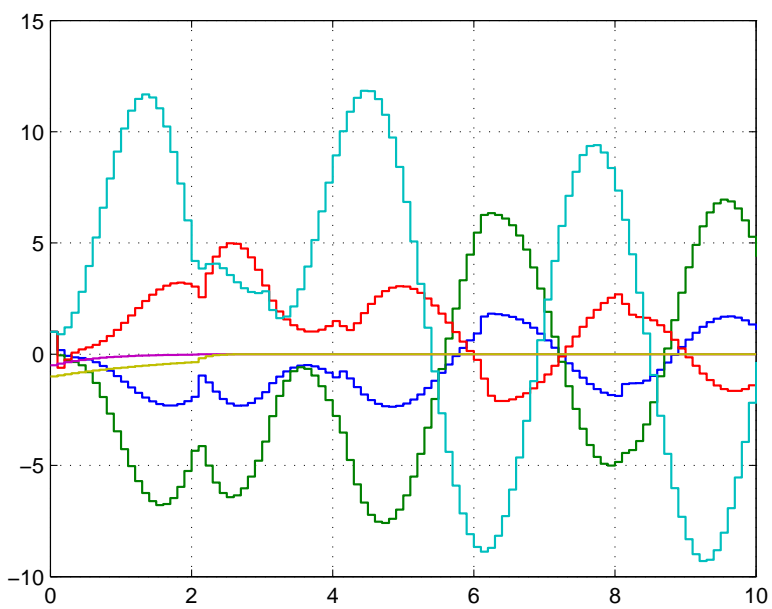

Figure 2: State $x$ of the compensated system with disturbance - amplitude vs. time (s) 


\section{Conclusions}

A methodology to solve different formulations of the disturbance decoupling problem, from the mere structural decoupling to the problem with state stability under arbitrary switching, so as to also guarantee local input-to-state stability of the compensated system, has been shown. The synthesis procedure has been supported by a complete computational framework, as was illustrated through the numerical example. The geometric approach, enriched with some new notions specifically addressed to switching linear systems, and linear matrix inequalities have provided the theoretical and computational background.

\section{References}

[1] W. M. Wonham, Linear Multivariable Control: A Geometric Approach, 3rd ed., SpringerVerlag, USA (1985), http://www.springer.com/us/book/9780387960715

[2] G. Basile and G. Marro, Controlled and Conditioned Invariants in Linear System Theory, Prentice Hall, USA (1992), http://www3.deis.unibo.it/Staff/FullProf/GiovanniMarro/gm_books.htm\#book5

[3] E. Zattoni, Self-bounded controlled invariant subspaces in measurable signal decoupling with stability: minimal-order feedforward solution, Kybernetika, 41, No. 1 (2005), 85-96, http://www.kybernetika.cz/view_file.html?item $=1925$

[4] G. Marro and E. Zattoni, "Self-bounded controlled invariant subspaces in model following by output feedback: minimal-order solution for nonminimum-phase systems," Journal of Optimization Theory and Applications, 125, No. 2 (2005), 209-229, DOI:10.1007/s10957004-1857-5.

[5] G. Marro, D. Prattichizzo, and E. Zattoni, A unified setting for decoupling with preview and fixed-lag smoothing in the geometric context, IEEE Transactions on Automatic Control, 51, No. 5 (2006), 809-813, DOI:10.1109/TAC.2006.875020.

[6] E. Zattoni, Decoupling of measurable signals via self-bounded controlled invariant subspaces: Minimal unassignable dynamics of feedforward units for prestabilized systems, IEEE Transactions on Automatic Control, 52, No. 1 (2007), 140-143, DOI:10.1109/TAC.2006.886499.

[7] A. Kaldmäe, Ü. Kotta, A. Shumsky, and A. Zhirabok, Measurement feedback disturbance decoupling in discrete-time nonlinear systems, Automatica, 49, No. 9 (2013), 2887-2891, DOI:10.1016/j.automatica.2013.06.013.

[8] A. M. Perdon and M. Anderlucci, Disturbance decoupling problem for a class of descriptor systems with delay via systems over rings, IMA Journal of Mathematical Control and Information, 27, No. 2, 4 (2010), 205-218, 405-418, DOI:10.1093/imamci/dnq008, 10.1093/imamci/dnq032.

[9] L. A. Marquez-Martinez and C. H. Moog, Disturbance decoupling for nonlinear timedelay systems, Asian Journal of Control, 9, No. 2 (2007), 190-194, DOI:10.1111/j.19346093.2007.tb00322.x. 
[10] A. Kaldmäe and C. H. Moog, Disturbance decoupling of time delay systems, Asian Journal of Control, 18, No. 3 (2016), 1130-1134, DOI:10.1002/asjc.1169.

[11] G. Stikkel, J. Bokor, and Z. Szabó, Disturbance decoupling problem with stability for LPV systems, in European Control Conference 2003, Cambridge, UK (2003), 558-563. http://ieeexplore.ieee.org/document/7085014/

[12] G. Conte, A. M. Perdon, and E. Zattoni, The disturbance decoupling problem with quadratic stability for LPV systems, in 1st IFAC Workshop on Linear Parameter Varying Systems, Grenoble, France, ser. IFAC-PapersOnLine, 48, No. 26 (2015), 1-6, DOI:10.1016/j.ifacol.2015.11.104.

[13] G. Conte, A. M. Perdon, and E. Zattoni, The disturbance decoupling problem for jumping hybrid systems, in 54th IEEE Conference on Decision and Control, Osaka, Japan (2015), 1589-1594, DOI:10.1109/CDC.2015.7402437.

[14] A. M. Perdon, E. Zattoni, and G. Conte, Disturbance decoupling with stability for linear impulsive systems, in 6th IFAC Symposium on System Structure and Control, Istanbul, Turkey, ser. IFAC-PapersOnLine, 49, No. 9 (2016), 1-6, DOI:10.1016/j.ifacol.2016.07.477.

[15] E. Yurtseven, W. P. M. H. Heemels, and M. K. Camlibel, Disturbance decoupling of switched linear systems, in 49th IEEE Conference on Decision and Control, Atlanta, GA (2010), 6475-6480, DOI:10.1109/CDC.2010.5717376.

[16] N. Otsuka, Disturbance decoupling with quadratic stability for switched linear systems, Systems \& Control Letters, 59, No. 6 (2010), 349-352, DOI:10.1016/j.sysconle.2010.03.010.

[17] E. Yurtseven, W. P. M. H. Heemels, and M. K. Camlibel, Disturbance decoupling of switched linear systems, Systems \& Control Letters, 61, No. 1 (2012), pp. 69-78, DOI:10.1016/j.sysconle.2011.09.021.

[18] E. Zattoni, Dynamic feedforward compensation of measurable signals in discrete-time linear switching systems, in 2013 American Control Conference, Washington DC (2013), 848-853, DOI:10.1109/ACC.2013.6579942.

[19] E. Zattoni and G. Marro, Measurable disturbance rejection with quadratic stability in continuous-time linear switching systems, in European Control Conference 2013, Zürich, Switzerland (2013), 2157-2162. http://ieeexplore.ieee.org/stamp/stamp.jsp?tp=\&arnumber $=6669248$

[20] E. Zattoni and G. Marro, A constructive condition for inaccessible signal rejection with quadratic stability in discrete-time linear switching systems, in 52nd IEEE Conference on Decision and Control, Florence, Italy (2013), 4650-4655, DOI:10.1109/CDC.2013.6760617.

[21] E. Zattoni, Measurable disturbance rejection with stability in continuous-time linear systems under dwell-time switching, in European Control Conference 2014, Strasbourg, France (2014), 2242-2247, DOI:10.1109/ECC.2014.6862247.

[22] E. Zattoni, A. M. Perdon, and G. Conte, Disturbance decoupling with stability in continuous-time switched linear systems under dwell-time switching, in 19th IFAC World Congress, Cape Town, South Africa, ser. IFAC Proceedings Volumes, 47, No. 3 (2014), 164-169, DOI:10.3182/20140824-6-ZA-1003.00908. 
[23] G. Conte, A. M. Perdon, and N. Otsuka, The disturbance decoupling problem with stability for switching dynamical systems, Systems \& Control Letters, 70, No. 8 (2014), 1-7, DOI:10.1016/j.sysconle.2014.05.002.

[24] E. Zattoni, A synthesis procedure for disturbance decoupling with local input-to-state stability in discrete-time switching linear systems, in 2015 American Control Conference, Chicago, IL (2015), 1071-1076, DOI:10.1109/ACC.2015.7170875.

[25] E. Zattoni, Stability issues in disturbance decoupling for switching linear systems, in 2015 SIAM Conference on Control and Its Applications, Paris, France (2015), 361-367, DOI:10.1137/1.9781611974072.50.

[26] E. Zattoni and G. Marro, Disturbance compensation in discrete-time switching linear systems subject to a dwell-time constraint, in 54th IEEE Conference on Decision and Control, Osaka, Japan (2015), 2307-2312, DOI:10.1109/CDC.2015.7402551.

[27] E. Zattoni, A. M. Perdon, and G. Conte, Disturbance decoupling with closed-loop modes stability in switched linear systems, IEEE Transactions on Automatic Control (Early View) (2016), DOI:10.1109/TAC.2015.2498123.

[28] E. Zattoni, A. M. Perdon, and G. Conte, Output-feedback model matching with strong stability in continuous-time switched linear systems, in 22nd Mediterranean Conference on Control and Automation, Palermo, Italy (2014), 525-530, DOI:10.1109/MED.2014.6961426.

[29] G. Conte, A. M. Perdon, and E. Zattoni, Model matching problems for switching linear systems, in 19th IFAC World Congress, Cape Town, South Africa, ser. IFAC Proceedings Volumes, 47, No. 3 (2014), 1501-1506, DOI:10.3182/20140824-6-ZA-1003.00162.

[30] E. Zattoni, Model matching by output dynamic feedback in discrete-time switching linear systems, in 54th IEEE Conference on Decision and Control, Osaka, Japan (2015), 23352340, DOI:10.1109/CDC.2015.7402556.

[31] A. M. Perdon, G. Conte, and E. Zattoni, Necessary and sufficient conditions for asymptotic model matching of switching linear systems, Automatica, 64, (2016) 294-304, DOI:10.1016/j.automatica.2015.11.017.

[32] Z. Sun and S. S. Ge, Stability Theory of Switched Dynamical Systems, ser. Communications and Control Engineering, Springer, USA (2011).

[33] Z. Sun and S. S. Ge, Switched Linear Systems: Control and Design, ser. Communications and Control Engineering. Springer, USA (2005).

[34] D. Liberzon, Switching in Systems and Control, ser. Systems \& Control: Foundations \& Applications. Birkhäuser, USA (2003).

[35] E. D. Sontag, Input to state stability: Basic concepts and results, in Nonlinear and Optimal Control Theory, ser. Lecture Notes in Mathematics, 1932, 163-220, P. Nistri and G. Stefani, Eds. Springer-Verlag, Germany (2008).

[36] E. D. Sontag and Y. Wang, New characterizations of input-to-state stability, IEEE Transactions on Automatic Control, 41, No. 9 (1996), 1283-1294, DOI:10.1109/9.536498.

[37] G. Basile and G. Marro, On the robust controlled invariant, Systems \& Control Letters, 9, No. 3 (1987), 191-195, DOI:10.1016/0167-6911(87)90039-9.

[38] H. Lin and P. J. Antsaklis, Stability and stabilizability of switched linear systems: A survey of recent results, IEEE Transactions on Automatic Control, 54, No. 2 (2009), 308-322, DOI:10.1109/TAC.2008.2012009. 
[39] S. Boyd, L. El Ghaoui, E. Feron, and V. Balakrishnan, Linear Matrix Inequalities in System and Control Theory. SIAM, USA (1994). 